\title{
Optical properties of antireflective flat or rough patterned topped silicon cones gratings
}

\section{Judikaël Le Rouzo ${ }^{1}$, Jean-Baptiste Brückner ${ }^{1}$, Abdelkerim Ferchichi ${ }^{2}$, Cécile Gourgon ${ }^{2}$, Gérard Berginc ${ }^{3}$ and Ludovic Escoubas*1}

\author{
${ }^{1}$ Aix Marseille Université, CNRS, Université de Toulon, IM2NP UMR 7334, 13397, Marseille, France \\ ${ }^{2}$ LTM, 17 Avenue des Martyrs, 38054, Grenoble cedex 9, France \\ ${ }^{3}$ Thales Optronique S.A., 78990, Elancourt, France
}

\begin{abstract}
Achieving a broadband antireflection property from material surfaces is one of the highest priorities in photosensing applications. To demonstrate a low reflectance, we are concerned with the study of the optical response of flat-, patterned- and rough-top silicon cone gratings. Based on previous

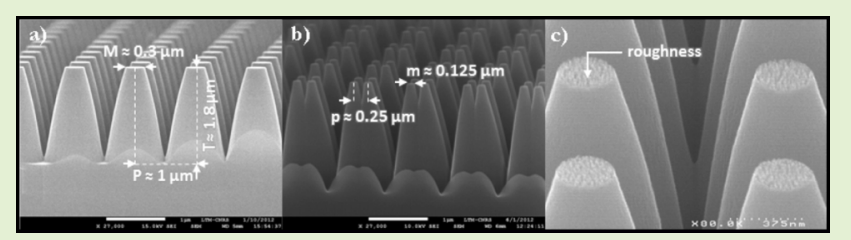
work exploring pyramid gratings, we first designed several flat top structures that theoretically demonstrate an antireflective character within the middle infrared region. In order to extend the antireflective properties up to the visible wavelengths, patterned- and rough-topped cones have been fabricated as well. Optical characterizations of several samples were carried out in the visible and mid infrared range, in order to determine the effect of structuration on the performances of the different cones gratings.
\end{abstract}

Keywords Diffraction gratings, Antireflection coatings, Silicon pyramidal structures, Scattering

Cite this article J. Le Rouzo, J.-B. Brückner, A. Ferchichi, C. Gourgon, G. Berginc and L. Escoubas: Adv. Device Mater., 2015, $1,23-26$

\section{Introduction}

Over the last few years, antireflective gratings have been widely investigated with the emergence of photovoltaics and photosensing. Reducing the reflectance of an incident radiation has become one of the highest priorities for such devices. Controlling the reflection of an incident radiation over an interface has then offered numerous applications for a wide range of domains. ${ }^{1}$ For instance, optical systems such as lenses need an antireflective coating to maximize their transmission coefficient. ${ }^{2}$ For a photodetector, reducing reflectance is an important matter, since the better the light penetrates into the material, the more it improves its sensitivity threshold. ${ }^{3}$ In photovoltaics, solar cells coated with an antireflective coating see their photon to electron conversion rate getting larger. Therefore, with the help of antireflective (AR) coatings, the efficiency of these devices has been greatly enhanced over the last few years. ${ }^{4-6}$ Concerning the stealth domain, reducing laser signature of a surface is an important matter as well. Nowadays, it is possible to distinguish two main categories of antireflective coatings: the optical thin films and the structured materials.

*Corresponding author, email ludovic.escoubas@univ-amu.fr
If the former one allow us to demonstrate complex optical function, ${ }^{7,8}$ thermal and mechanical issues have restricted the number of applications. Nevertheless, with the advent of nanophotonics, it has been possible to reduce the phenomenon of light reflection through direct structuring in the material. ${ }^{9-13}$ Indeed, as the observation of the surface of a moth eye unveiled a regular arrangement of $200 \mathrm{~nm}$ cones responsible for a large reflectance reduction, ${ }^{14}$ one tried to mimic such antireflective properties by structuring the surface of materials. Thanks to the nanofabrication technology advances, different surface patterns have been designed to achieve low reflectance over definite spectral bands. It can be cited shapes such as wires or cones leading to broad antireflective bands at different regime following the dimensions of the structures. Random structuration has demonstrated a strong efficiency due to light scattering and light trapping along the rough surface. ${ }^{15-18}$ In the visible domain, reflectance can be easily reduced under the percent from random structuration of the surface of a silicon wafer. ${ }^{16}$ However, the antireflective spectral band of randomly structurated surfaces cannot easily be tuned and the various accurate modeling techniques established ${ }^{19-21}$ have not proven yet any rigorous prediction of the optical properties such as specular and scattered reflectances of such surfaces, especially in the case of high roughness. On the contrary, 

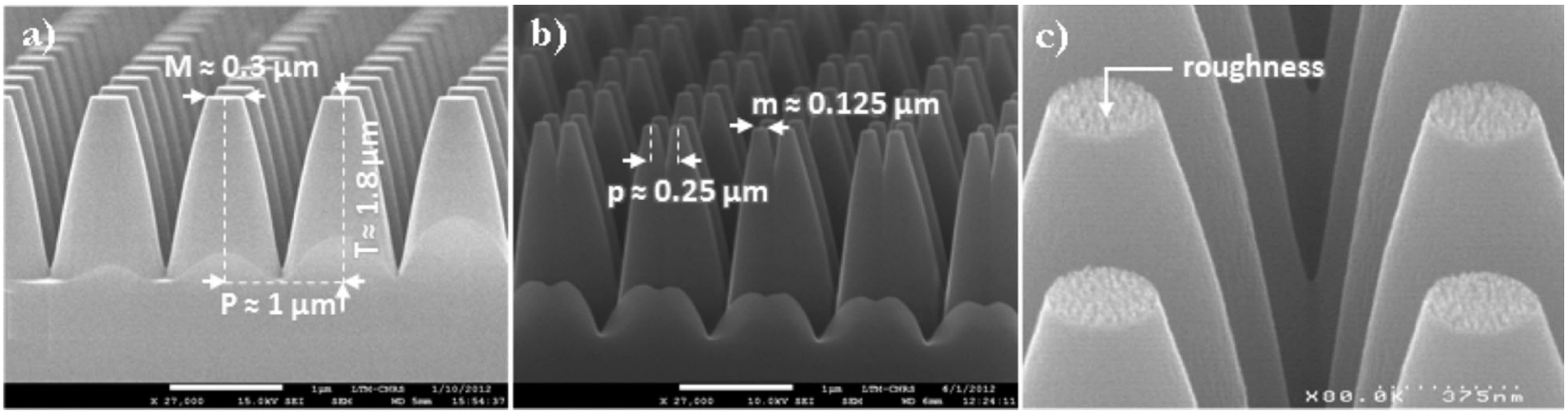

Figure 1 Images and geometric parameters of $a$ flat-topped, $b$ patterned-topped and $c$ rough-topped silicon cones gratings

periodic structures can be easily modeled with numerical methods such as FDTD $^{22}$ or RCWA. ${ }^{23}$ Furthermore, their antireflective spectral bands can be tuned as well through adjusting the period of the subwavelength patterns whereas the height of the patterns is influencing the reflectance of the surface. ${ }^{24-28}$ The principle objective here is to demonstrate an antireflective (AR) coating with reflectance less than $2 \%$ for specific spectral bands, where the atmospheric transmission is important: the $0.7-2.5$ and $3-5 \mu \mathrm{m}$ bands. The $8-12 \mu \mathrm{m}$ region is not treated though. Based on previous numerical analysis on pyramid gratings, ${ }^{29}$ we have fabricated three different antireflective gratings: flat-, patterned- and rough-topped silicon cones. The point here is to compare the performances of these fabricated gratings in the visible $(\lambda=0.4-0.8 \mu \mathrm{m})$ and the mid infrared range $(\lambda=3-5 \mu \mathrm{m})$. Theoretical studies on truncated silicon cone gratings have permitted to show a reflectance less than $2 \%$ in the $\lambda=3-5 \mu \mathrm{m}$ region. Moreover, photonic band dispersion diagrams of these structures highlighted the importance of the top flat. ${ }^{30}$ Indeed in a photonic crystal, light can be coupled in the form of Bloch modes. The concept of the so called light line is very useful for structures with $2 \mathrm{D}$ mode confinement to divide a photonic band dispersion diagram into a region of a non-guided continuum of modes, a region of discrete guided modes and a forbidden region, where no propagating modes can exist. ${ }^{31}$ Indeed, it has been understood that the top flat created a discontinuity in the refractive index profile which moves the surface mode of a standard silicon cone grating under the light line when a flat is present on the top of the silicon cone. Those modes located under that line would couple with the photonic structure, thus decreasing the amount of the energy reflected in the incident medium. However, the top flat length had to be wisely chosen since there is a compromise: a top flat is required for surface mode coupling but the larger the top flat, the higher the reflectance is. It has also been demonstrated that if pyramid structures of period $P$ exhibit antireflective properties centered at the wavelength $\lambda$, pyramid structures of period $P / k$ exhibit antireflective properties at the wavelength $\lambda / k$ (where $k$ is a real called 'scale factor'). Therefore, by applying a scale factor ' $k$ ', smaller pyramids can be efficient at shorter wavelengths. Thus, adding on the top of the former structures, smaller pyramids dimensioned to be antireflective in the visible, one can widened the antireflective band. ${ }^{32}$ Following this principle, broad antireflective band operating from the visible up to the infrared region, have been fabricated..$^{29}$ However, it has been observed that the AR efficiency in the middle infrared region was slightly degraded due to the patterning. Therefore, we have been thinking of carrying out a third type of grating made of rough-topped truncated pyramids.

\section{Experimental methods}

The general concept is to combine the antireflective effect of periodic structurations, which can be adjusted by the scale factor ' $k$ ' to be efficient in the mid IR range, with a rough surface scattering the visible light along the surface. However, as it has been said in introduction, no rigorous method is available to calculate the reflectance of such rough structure. Indeed, the shape of this rough surface is outside the validity domain of the classical approximated methods such as small slope approximation. ${ }^{19-21}$ Therefore, these structures have been directly fabricated, without other calculations than those performed for the flat-topped pyramids. ${ }^{29}$ Geometric parameters are kept the same: the base period $\mathrm{P}$ of the pyramid is $1 \mu \mathrm{m}$ long, height $\mathrm{T}$ of the pyramid is of $1.8 \mu \mathrm{m}$, top flat length $M$ is of $0.32 \mu \mathrm{m}$. There are no spacings in between the structures. For patternedtopped structures, the geometrical parameters of the smaller pyramids follow a ratio of $M / 2 P$. Several plasma etching with controlled slope processes have been performed in order to carry out the structures over a large surface. Concerning the rough-topped pyramids, a first photolithography followed by a plasma etching has been performed to obtain the periodic patterns, which are cone gratings instead of pyramid gratings (due to the poor resolution of the photolithography equipment). Then, a second selective

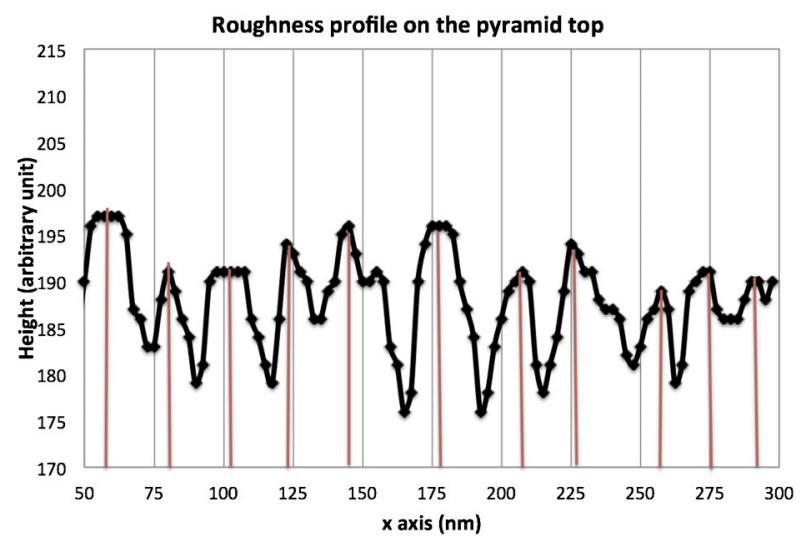

Figure 2 Roughness profile on pyramid top 

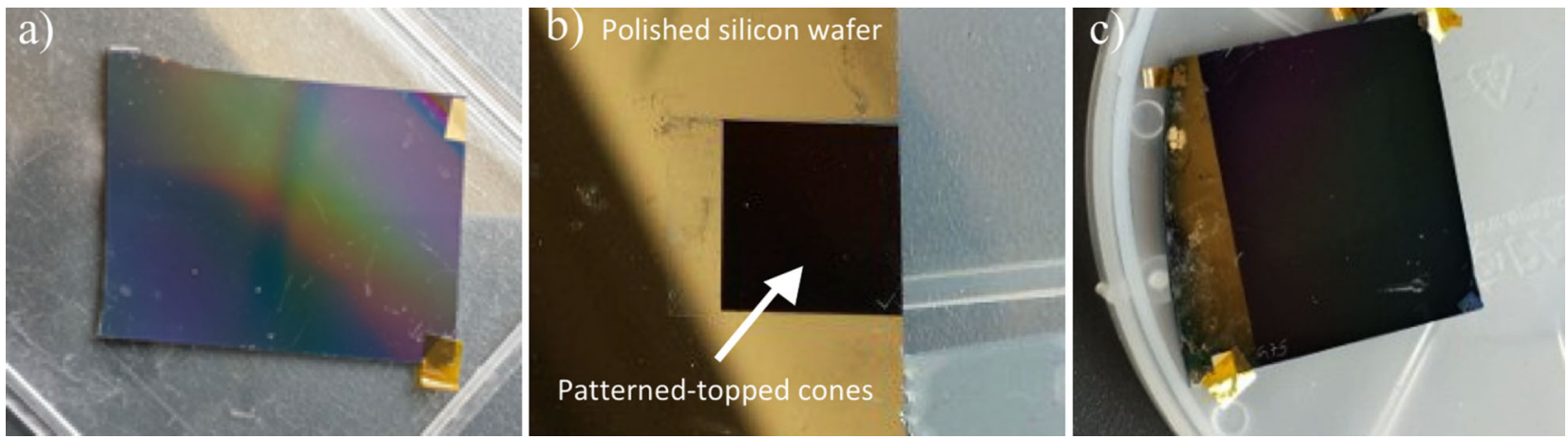

Figure 3 Photographs of $a$ flat-topped, $b$ patterned-topped and $c$ rough-topped silicon cones gratings

plasma etching step is simply added, without any photolithographic step before, to allow roughness to be obtained on the flat top. Remarkable V-shaped structures have been achieved, without any spacing in between, and presenting geometrical parameters very close to those specified (Figs. 1-3). From Fig. 1c, the mean roughness of the cone top was estimated to be $30 \pm 5 \mathrm{~nm}$. Furthermore, the SEM image of Fig. $1 c$ allows obtaining the roughness profile of Fig. 2 (extracted from one line on one cone top). From this profile, the mean distance between the peaks was estimated to be $25 \pm 5 \mathrm{~nm}$.

\section{Results and discussion}

A first look at the different samples (Fig. 3) can give a hint to the antireflective properties of the samples in the visible domain. Indeed, a polished silicon wafer generally presents a grey surface with a reflectance of the order of $40 \%$ in the visible domain. The first fabricated grating, the top flat cone grating, demonstrates a dark surface (Fig. 3a), letting appear though the typical colored stripes of diffraction gratings. Following certain applications, especially the imaging ones, we may want to reduce at possible these diffracted orders that could lead to cross talking effects in CMOS imagers, for instance. Meanwhile, patterned- and rough-topped cones display an even darker surface (Fig. $3 b$ and c), indicating that the antireflective potential has been improved in the visible range. To confirm this observation, optical performances of the different types of gratings were then investigated in the visible range (Fig. $4 a$ ) using an integrating sphere and in the infrared region (Fig. 4b) with a Fourier Transform Infrared spectrometer. The flat-topped cones have been formerly designed to show antireflective properties only in the $\lambda=3-$ $5 \mu \mathrm{m}$ region. ${ }^{24,30}$ However, integrating sphere measurements show us that these gratings can demonstrate as well a very low reflectance in the visible region. Indeed, an average reflectance of $2.5 \%$ has been achieved in the $\lambda=0.45-$ $0.75 \mu \mathrm{m}$ range (Fig. $4 a$ ). Furthermore these measurements clearly demonstrates that the AR properties are effectively enhanced by the structuration of the top flat (patterned- or rough-topped cones), as for these gratings, the average reflectance in the visible range has been reduced under the percent. On the one hand, in the visible range, the patterned-topped cones creates a discontinuity in the refractive index profile and the mechanisms reducing the reflectance is the same as the one of top-flat cones in the IR wavelength range. On the other hand, for rough-topped cones, the reflectance in the visible range is decreased because the roughness creates an effective medium which refractive index is intermediate between silicon and air refractive indices. As expected, the FTIR characterizations of the flat-topped cones in the middle infrared show that a reflectance less-than-2\% has been achieved (Fig. 4b). These measurements also confirm that the reflectance is slightly degraded by the patterns on the top of the cones. However, as for the flat-topped cones, the rough-topped one display a less than $2 \%$ reflectance. This can be explained by the fact that the rough surface is somewhat very close to the flat one in its shape. Indeed, as said before, the mean roughness is
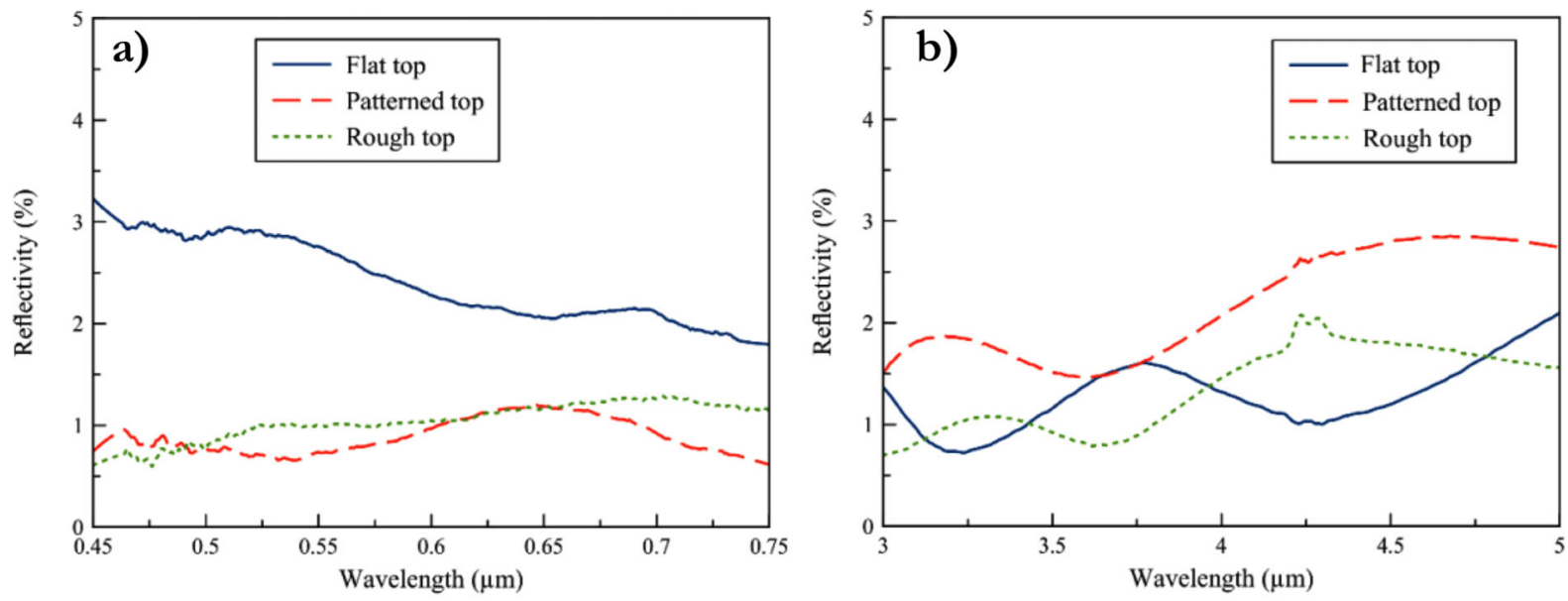

Figure 4 a Integrating sphere and $b$ FTIR spectrometry measurements 
estimated to be $30 \pm 5 \mathrm{~nm}$, which is very small when compared to the wavelength $(\sim \lambda / 100)$. Thus, the discontinuity in the refractive index profile that moves the surface mode of a standard silicon cone grating under the light line and reduces the reflectance in this wavelength range (3$5 \mu \mathrm{m})$, remains the same for flat- or rough-topped cones. On the other hand, the structures of the patterned-topped cones were slightly too large $(p=0.25 \mu \mathrm{m}$ and $m=0.125 \mu \mathrm{m})$ when compared to the wavelength $(\sim \lambda / 10)$ to reach the same discontinuity in the refractive index profile, thus explaining the higher average reflectance $(R \approx 2.5 \%)$.

\section{Conclusions}

Finally, after having investigated several types of antireflective structures, we have fabricated different truncated cones gratings displaying remarkable antireflective properties into the visible and mid infrared region. If the structuration of the flat top was critical to enhance the $A R$ properties in the visible, only rough-topped cones can demonstrate a less than $2 \%$ reflectance in both visible and mid infrared ranges. In the future, we may consider of adding a third level of periodicity in order to extend the AR band, or finding different patterns for the top-flat or to fabricate entirely rough cones as well.

\section{Conflicts of interest}

The authors declare that there is no conflict of interest with the ANR in the N-POEM project (see "Acknowledgements").

\section{Acknowledgements}

The authors gratefully acknowledge the financial support from 'Agence Nationale de la Recherche' (ANR) for funding the N-POEM project.

\section{References}

1. S. Chattopadhyay, Y. F. Huang, Y. J. Jen, A. Ganguly, K. H. Chen and L. C. Chen: Mater. Sci. Eng. R: Rep., 2010, 69, 1-35.

2. K. Baker: Appl. Opt., 1999, 38, 352-356.

3. D. Braun: Appl. Opt., 1988, 27, 2006-2011.

4. J. Chen, C. Con, M. Yu, B. Cui and K. Sun: ACS Appl. Mater. Interf., 2013, 5, 7552-7558.
5. K. Park, Z. Guo, H. Um, J. Jung, J. M. Yang, S. K. Lim, Y. S. Kim and J. Lee: Opt. Expr., 2010, 19, A41-50.

6. K. Forberich, G. Dennler and M. C. Scharber: Thin Solid Films, 2008, 516, 7167-7170.

7. H. A. MacLeod: in 'Thin-film optical filters', 668; 2010, T. \& Francis Ed. IV.

8. M. Mazur, D. Wojcieszak, J. Domaradzki, D. Kaczmarek, S. Song and F. Placido: Opto-electr. Rev., 2013, 21, 223-228.

9. C. Yeo, J. Kim, Y. Song and Y. Lee: Nanoscale Res. Lett., 2013, 8, 159.

10. H. Lin, W. Chen, C. Wang and F. Hong: Thin Solid Films, 2013, 529, 138-142.

11. T. H. Her, R. J. Finlay, C. Wu and E. Mazur: Applied Physics A: Materials Science and Processing, 2000, 70, 383-385.

12. J. B. Brückner, J. Le Rouzo, L. Escoubas, G. Berginc, O. Calvo-Perez, N. Vukadinovic and F. Flory: Opt. Expr., 2013, 21, 16992-17006.

13. J. B. Brückner, V. Brissonneau, J. Le Rouzo, A. K. Ferchichi, C. Gourgon, C. Dubarry, G. Berginc and L. Escoubas: Appl. Phys. Lett., 2014, 104, 081114-081118.

14. P. B. Clapham and M. C. Hutley: Nature, 1973, 244, 281-282.

15. S. K. Srivastava, D. Kumar and K. Singh: Solar Energy Mater. Solar Cells, 2010, 94, 1506-1511.

16. J. Zhou, M. Hildebrandt and M. Lu: J. Appl. Phys., 2011, 109, 053513.

17. N. Vourdas, K. Dalamagdikis, I. Kostis, M. Vasilopoulou and D. Davazoglou: Opt. Commun., 2012, 285, 5229-5234.

18. D. Maestre, J. Le Rouzo, D. Barakel, O. Palais, L. Escoubas, C. Alfonso and L. Charrin: 'Growth and characterization of Si nanowires by an electroless etching process', Proc. 24th Eur. Photovoltaic Solar Energy Conf., 2009, 408-410.

19. G. Berginc: 'Small-amplitude perturbation theory for two-dimensional surfaces', in 'Light scattering and nanoscale surface roughness', (ed. A. A. Maradudin); 2007, Berlin, Springer.

20. G. Berginc: Progr. Electromagn. Res., 2002, 37, 251-287.

21. G. Berginc and C. Bourrely: Progr. Electromagn. Res., 2007, 73, 131211.

22. F. L. Texeira: Appl. Comput. Electromagn. Soc. J., 2010, 25, 1-14.

23. L. Lifeng, J. Chandezon, G. Granet and J. Pierre: Appl. Opt., 1999, 38, 304-313.

24. L. Escoubas, J. J. Simon, M. Loli, G. Berginc, F. Flory and H. Giovannini: Optics Commun., 2003, 226, 81-88.

25. H. Toyota, K. Takahara, M. Okano, T. Yotsuya and H. Kikuta: Jpn J. Appl. Phys. 2: Lett., 2001, 40, L747-L749.

26. K. Shoo, Y. Li and E. Chang: Comput. Phys. Commun., 2009, 180, 1721-1729.

27. H. Wang, T. Tsai, K. Lai, T. Wei, Y. Wang and J. He: Opt. Expr., 2012, 20, A94-A103.

28. R. Bouffaron, L. Escoubas, V. Brissonneau, J. J. Simon, G. Berginc, P. Torchio, F. Flory and P. Masclet: Opt. Expr., 2009, 17, 21590-21597.

29. J. B. Brückner, J. Le Rouzo, L. Escoubas, G. Berginc, C. Gourgon, O. Desplats and J. J. Simon: Opt. Expr., 2013, 21, 16043-16055.

30. R. Bouffaron, L. Escoubas, J. J. Simon, P. Torchio, F. Flory, G. Berginc and P. Masclet: Opt. Expr., 2008, 16, 19304-19309.

31. P. Kaspar, R. Kappeler, D. Erni and H. Jäckel: Opt. Expr., 2011, 19, 24344-24353.

32. L. Escoubas, R. Bouffaron, V. Brissonneau, J. J. Simon, G. Berginc, F. Flory and P. Torchio: Opt. Lett., 2010, 35, 1455-1457. 